$\pm N / E$

Global burnals Inc.

8

\title{
Pharmaceutical Drugs and the Human Energy System (Biofield)
}

By Seema Bhattessa

Abstract- What is Human Life-Force Energy and What Type of Proof Do We Have?: The concept of subtle human energy fields, or life-force energy, has been recognised and woven into traditional healing systems for millennia. Traditional Chinese Medicine (TCM) describes an intricate network of energy meridians through which this energy, known as "chi" circulates. And, in the traditional Indian system of Ayurveda, the human energy field takes the form of energy vortexes called "chakras", through which energy, known as "prana" travels.

Accumulating evidence for the existence of these and other subtle, spatially-oriented and biologically-generated, human energy fields has been demonstrated through objective testing methods. As a result, in 1992, the term "biofield" emerged to describe this energy. Biofield Energy is defined as "a massless field, not necessarily electromagnetic, that surrounds and permeates living bodies and affects the body."[1]

Keywords: biofield; biofield science; biofield research; drugs; pharmaceutical drugs; prescription medicine; human energy system; energy flow; manipulation of the biofield.

GJMR-B Classification: NLMC Code: QV 55

Strictly as per the compliance and regulations of:

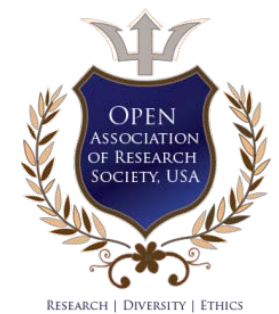

(C) 2020. Seema Bhattessa. This is a research/review paper, distributed under the terms of the Creative Commons AttributionNoncommercial 3.0 Unported License http://creativecommons.org/licenses/by-nc/3.0/), permitting all non-commercial use, distribution, and reproduction in any medium, provided the original work is properly cited. 


\title{
Pharmaceutical Drugs and the Human Energy System (Biofield)
}

\author{
Seema Bhattessa
}

\begin{abstract}
What is Human Life-Force Energy and What Type of Proof Do We Have?: The concept of subtle human energy fields, or life-force energy, has been recognised and woven into traditional healing systems for millennia. Traditional Chinese Medicine (TCM) describes an intricate network of energy meridians through which this energy, known as "chi" circulates. And, in the traditional Indian system of Ayurveda, the human energy field takes the form of energy vortexes called "chakras", through which energy, known as "prana" travels.
\end{abstract}

Accumulating evidence for the existence of these and other subtle, spatially-oriented and biologically-generated, human energy fields has been demonstrated through objective testing methods. As a result, in 1992, the term "biofield" emerged to describe this energy. Biofield Energy is defined as "a massless field, not necessarily electromagnetic, that surrounds and permeates living bodies and affects the body."[1]

Biofields are thought to function as a communication conduit that formats and relays information between physiological systems and enables near-instantaneous response times.[2] This inclusive approach affirms core concepts within traditional healing systems, while inviting a more expansive model of living systems than is currently offered by biological science.[3] Through the biofield concept, biological systems are integrated both internally and within their environments across time and space. These energetic relationships increase in scale from subatomic and organismic, to interpersonal and cosmic levels.[2] Potential evidence of the latter can be found in the correlation between solar storms and increased rates and severity of myocardial infarctions. [1,4]

Keywords: biofield; biofield science; biofield research; drugs; pharmaceutical drugs; prescription medicine; human energy system; energy flow; manipulation of the biofield.

\section{What is Human Life-Force Energy and What Type of Proof do We have?}

T he concept of subtle human energy fields, or lifeforce energy, has been recognised and woven into traditional healing systems for millennia. Traditional Chinese Medicine (TCM) describes an intricate network of energy meridians through which this energy, known as "chi" circulates. And, in the traditional Indian system of Ayurveda, the human energy field takes the form of energy vortexes called "chakras", through which energy, known as "prana" travels.

Author: Restoring Therapies, London, United Kingdom. e-mail:sb.3research@gmail.com
Accumulating evidence for the existence of these and other subtle, spatially-oriented and biologically-generated, human energy fields has been demonstrated through objective testing methods. As a result, in 1992, the term "biofield" emerged to describe this energy. Biofield Energy is defined as "a massless field, not necessarily electromagnetic, that surrounds and permeates living bodies and affects the body." [1]

Biofields are thought to function as a communication conduit that formats and relays information between physiological systems and enables near-instantaneous response times. [2] This inclusive approach affirms core concepts within traditional healing systems, while inviting a more expansive model of living systems than is currently offered by biological science. [3] Through the biofield concept, biological systems are integrated both internally and within their environments across time and space. These energetic relationships increase in scale from subatomic and organismic, to interpersonal and cosmic levels.[2] Potential evidence of the latter can be found in the correlation between solar storms and increased rates and severity of myocardial infarctions..$^{[1,4]}$

Several distinct biofields have been identified, some of which have been exploited for diagnostic and therapeutic uses, and more are being posited. A few of these include:

\section{a) Electromagnetic Fields}

One of only 4 forces in nature - along with gravity, and strong and weak nuclear forces, electromagnetism is the most important natural force at work in living systems. ${ }^{[1]}$ Electromagnetic fields (EMFs) generate electrocardiograms and electroencephalograms that have been a standard of medical diagnostics for decades. More recently, biofield investigations show that subtle magnetic fields generated by the heart radiate outwards from the body. This energy is transmissible to nearby individuals, producing a synchronisation of EEG waves between the source and recipient. ${ }^{[1,2]}$

\section{b) Biophotons}

Also known as ultraweak photon emissions (UPE), cellular glow, or ultraweak bioluminescence, this biofield energy can be detected both in cell cultures and on the body surface via photomultipliers. ${ }^{[4]}$

Biophoton generation patterns have been associated with fluctuations in cerebral blood flow and 
metabolism. ${ }^{[2]}$ High levels of UPEs have been measured from reactive oxygen species indicating that biophotons may prove to be a sensitive marker for metabolic stress. ${ }^{[2]}$

\section{c) Cellular Membrane Potentials and Charged Particles}

Taken together, the combined effects of transmembrane potentials with the oscillatory frequencies of molecules, and charged particles across an entire organism, create what has been described as a 'bioelectric scaffold' through which stem cell development, tissue repair, organ regeneration, and homeostatic processes are facilitated and coordinated. ${ }^{[1,2,5]}$

\section{d) Sub-EMF Level Electron Flux Fields}

The possibility of a sub-electromagnetic energy field has been raised via experimental evidence, which may support the phantom limb phenomenon reported by amputees. The evidence stems from energy imaging in plants, which confirms that when leaves are cut the energy of the cut portion is retained as a precise and accurate phantom. ${ }^{[4]}$ Moreover, leaf phantoms conduct electrical current and interact with electromagnetic fields. ${ }^{[4]}$

\section{e) Biofield Receptor Systems}

Where there is an effector there is a receptor, and the search for biofield receptor systems to date, has produced evidence for three such categories ${ }^{[2]}$ :

1. Molecular-level

- DNA transcription is modulated by low-frequency EMFs. ${ }^{[2]}$

2. Charge flux sites

- Voltage-gated calcium channels are affected by the presence of low-frequency EMFs. ${ }^{[2]}$

3. Endogenous EMFs

- Gap junctions constitute a bioelectric intracellular communication system that guides tissue development and maintenance. ${ }^{[2]}$

Additionally, a fourth proposed concept for a biofield receptor system integrates all of the above, and revolves around the fascial network of collagen fibres and water molecules, that surrounds organs and tissues. Collagen fibres are capable of conducting and modifying photon pulses and provide a form of surveillance, alongside the immune and nervous systems. Water molecules contribute to this receptor system via local synchronisation of electron spin, causing the collagen matrix in that area to act as a frequency-specific electromagnetic receptor system. ${ }^{[1,2,4,6]}$

\section{f) Clinical Evidence of Biofields}

In vitro and in vivo published studies dating back to the 1960s demonstrated the ability of biofield energy to facilitate wound healing and inhibit cancer cell viability. ${ }^{[7,8]}$ More recently, an abundance of clinical data provides illuminating (and increasingly irrefutable) evidence for the existence of human biofield energy, such as the following reports:

- An intriguing 2015 study reported that sceptical volunteers given a "crash course" in energy healing cured laboratory mice injected with lethal doses of cancer cells. ${ }^{[7]}$

- In a 2016 experiment, Korean scientists produced demonstrable evidence for the existence of acupuncture points. In that study, dye injected into acupuncture points formed visible lines, while the same effect was not observed at non-acupuncture points. ${ }^{\left[{ }^{[}\right]}$

- A newly developed biofield device, which delivers direct current through a saline water immersion foot bath, produced improvements in red blood cell morphology and CO2 transport in healthy participants in a 2018 study. ${ }^{[10]}$

- To date, over 15 clinical trials have been conducted on various biofield therapies for cancer patients, with reports of reduced pain and fatigue and improvements in biomarkers. ${ }^{[11]}$

g) Intrinsic and Extrinsic Factors that Affect the Human Energy System

The energy values of substances both intrinsic and extrinsic to the human body have varying effects on biofield energy. These include frequencies we generate ourselves, such as our thoughts and emotions, energy from substances we intentionally bring into our own personal biofields, and natural and manmade energy fields in our environment that affect us energetically in a multitude of ways.

Basic nutritional elements have been shown to shift the present energy within the body as they are absorbed and assimilated. Electrical conductance on the skin surface at acupuncture points has been shown to shift in response to glucose ingestion, revealing a fluctuating pattern of energy shifting through the meridians, as glucose is ingested and assimilated. ${ }^{[12]}$

Thoughts, i.e. the electrical activity of cognitive function, affect the central and autonomic nervous systems, contributing to the unique electromagnetic field of an individual, or aura. The aura radiates outwards, carrying with it information about physiological, mental, and emotional status of the individual. ${ }^{[13]}$

h) Vibratory Signatures and their Effects on the Biofield

All living and non-living things have a unique vibratory rate that can be measured and quantified in Hertz $(\mathrm{Hz})$. Living beings have higher rates of vibration while non living objects have little to no vibratory rate.

The foods we consume contribute their own inherent rates of vibration to our own, raising or lowering the overall rate accordingly. In general, highly 
processed, pesticide-laden foods have relatively lower vibratory rates, whilst fresh, organic plant foods, and fermented and raw sprouted foods offer the highest rates. $^{[14,15]}$

Similarly, light energy in the visible spectrum is thought to exert a range of effects based on the vibratory rate of each colour. In Ayurveda, colours within the lower frequency ranges correlate to lower chakras, and influence functions such as survival and sexuality, while higher frequency colours correlate to higher functions: communication, love, intuition, and spirituality. ${ }^{[16]}$

\section{i) Energetic Manipulation of Biofields}

Numerous therapeutic systems and medical practices have been found to have a beneficial influence on human biofields, while other forms of energy have notably detrimental or even mixed effects.

\section{Examples include:}

\section{- Aromatherapy}

Plant essential oils have among the highest recorded vibrational rates. Oils at the lower end of the range are used to heal physical illnesses, those in the middle of the range support emotional health, and those in the upper-frequency ranges are thought to promote spiritual growth. ${ }^{[17]}$

- Grounding

This is a practise that uses the Earth's strong negative charge to supply electrons to the body. Grounding is practised by placing one's bare feet on the ground, and is thought to neutralise free radicals, in essence, acting as an antioxidant. ${ }^{[18]}$ Published research has reported improvements in sleep, pain, stress response, immune function, and blood sugar regulation. ${ }^{[6]}$

\section{- Pulsed EMF}

Embryonic development, tissue regeneration, and other processes requiring biological organisation are thought to occur through energy field phenomena. Pulsed EMF therapy has been used to regenerate limbs in salamanders, and is being investigated as a potential treatment for healing bone fractures. ${ }^{[2]}$

\section{- Wireless Technology}

Counter to prevailing public health doctrine, detrimental non-thermal health effects of nonionising EMFs from cell phones and other wireless technology have been well-documented in the literature. ${ }^{[1,2]}$ Studies show neurodevelopmental, neurodegenerative, cognitive, behavioural, endocrine, immune, and genotoxic effects. ${ }^{[19]}$ The pervasive use of wireless devices, and the imminent deployment of $5 \mathrm{G}$, raise concerns about the effects of our increasingly electromagnetic environment on the health of humans and the environment as a whole. ${ }^{[20]}$

\section{- Prescription Medicines}

Pharmaceutical drugs interact in various ways with biofields, some beneficial and others harmful.

o On the potentially beneficial side, certain drugs have been found to act synergistically with bio-magnetic fields, increasing response to drugs by severalfold. ${ }^{[20]}$ Drug delivery systems are being developed around this effect using magneto-electric nanoparticles designed to manipulate membrane electric fields to improve absorption- a process known as electroporation. ${ }^{[21]}$ Currently, however; though highly effective, its use is limited by the inability of cells to reverse the process and reseal the cell membrane. ${ }^{[22]}$

- Pharmaceuticals may also exert a range of adverse mental and emotional effects through their interactions with the biofield. Empirical reports exist in which patients describe shifts of consciousness from taking certain prescription medications; in some instances, a fragmented, disconnected sense of self and in others an ecstatic sense of unity and wholeness, either scenario being an unsettling departure from reality. Attempts to recreate such sensations have pointed toward over-inhibition or over-activation of the anterior insula which serves an important role in maintaining an individual's sense of self. ${ }^{[23]}$

\section{j) My Professional Experience and a Call to Action}

In my professional experience as a pharmacist and holistic energy therapist, I have observed, at times, the biofield effects of pharmaceutical drugs in my patients. This has occurred most notably in patients on hormone replacement, or other endocrine therapies, in the form of a certain sluggishness in chakra energy. In these instances, as well as where the source stems from other types of physical, mental, or emotional trauma, working with the client's energy field often helps improve the flow of stagnant energy, heal auric tears and remove any blockages that may have developed as a result of health issues, stress and anxiety, depression and other life experiences.

Restoring the balance and the flow of the energy system is a subtle healing process that is not always obvious, but often recognised by others, and expressed in the way that they respond towards you. I have also used energy therapy to help strengthen a client's vulnerability to adverse situations, or relations with others, in the workplace and at home. In this way, the energy system affects, and is affected by, our behaviour. Biofield therapeutics can act as an early intervention for behavioural health, a self-help tool, and a social investment.

Biofield science is growing rapidly, particularly in the fields of psychiatric and neurodegenerative disorders. However, funding usually favours industry applications, and is lacking in basic science and clinical 
applications research. ${ }^{[2]}$ Amongst the most urgent areas requiring further study, researchers have identified the lack of information on the effects of dose, delivery mode, and type of therapy on clinical outcomes. ${ }^{[11]}$ With this brief synopsis on biofield science, I invite my colleagues within the medical, health, and energyhealing spheres to further investigate these important topics. I am seeking to collaborate directly with interested professionals, and to conduct and publish studies that will contribute to the growing body of knowledge about the human energy field.

Conflict of Interest of interest.

The author(s) declare that they have no conflict

\section{References Références Referencias}

1. Biofield Science and Healing: History, Terminology, and Concepts. Global Advances in Health and Medicine, 2015 https://journals.sagepub.com/doi/ pdf/10.7453/gahmj.2015.038.suppl

2. Biofield Physiology: Framework for an emerging discipline. Global Advances in Health and Medicine, 2015 . https://journals.sagepub.com/doi/pdf/ 10.7453/gahmj.2015.015.suppl

3. Biofield Science and Healing: An Emerging Frontier in Medicine. Global Advances in Health and Medicine 2015 https://journals.sagepub.com/doi/ pdf/10.7453/gahmj.2015.106.suppl

4. Biofield Science: Current Physics Perspectives Global Advances in Health and Medicine, 2015 https://journals.sagepub.com/doi/pdf/10.7453/gah mj.2015.011.suppl

5. Electromagnetic homeostasis and the role of lowamplitude electromagnetic fields on life organization. Electromagnetic Biology and Medicine 2017. Volume 36(2) https://www.tandfonline.com/ doi/full/10.1080/15368378.2016.1194293?scroll=top \&needAccess $=$ true

6. An Overview of Biofield Devices. Global Advances in Health and Medicine, 2015 https://journals.sage pub.com/doi/pdf/10.7453/gahmj.2015.022.suppl

7. Challenges for Preclinical Investigations of Human biofield Modalities. Global Advances in Health and Medicine, 2015 https://journals.sagepub.com/ doi/pdf/10.7453/gahmj.2015.013.suppl

8. Adee, S. Our bodies are full of electricity that could help us fight cancer. May 30, 2019; Available from: https://qz.com/1630159/bioelectricity-may-be-keyto-fighting-cancer/.

9. ReShel, A. Science Finally Proves Meridians Exist. April 20th, 2016; Available from: https:// upliftconnect.com/science-proves-meridians-exist/.

10. Bio-field array: a dielectrophoretic electromagnetic toroidal excitation to restore and maintain the golden ratio in human erythrocytes. Physiological
Reports, 2018 https://physoc.onlinelibrary.wiley. com/doi/pdf/10.14814/phy2.13722

11. Clinical Studies of Biofield Therapies: Summary, Methodological Challenges, and Recommendations. Global Advances in Health and Medicine, 2015 https://journals.sagepub.com/ doi/pdf/10.7453/gahmj.2015.034.suppl

12. Different patterns of dynamic variations on electrical conductances of acupoints between Qi Vacuity and Qi non-Vacuity after glucose ingestion. J Altern Complement Med, 2011. 17(9): p. 843-9 https://www.ncbi.nlm.nih.gov/pubmed/21854198

13. Monitoring the Human Health by Measuring the Biofield "Aura": An Overview. International Journal of Applied Engineering Research, 2015. Vol. 10 No.35 https://www.academia.edu/13121004/Monitoring_th e_Human_Health_by_Measuring_the_Biofield_Aura An_Overview

14. High Vibrational Foods: How to Raise Your Consciousness with Your Diet. October 20, 2014; Available from: http://www.thehealersjournal.com/ 2014/10/20/highvibrational-foods-howto-raise-yourconsciousness-with-your-diet/.

15. Benardis, M., Eating High-Vibrational Foods for Good Health and Longevity.

16. USING COLORS TO CREATE YOUR REALITY. Available from: https://raiseyourvibrationtoday. com/using-colors-to-create-your-reality/.

17. Higley, C.A., Reference Guide to Essential Oils 2018.

18. Mercola, D. How Your Body Generates Electricity. March 01, 2014; Available from: https://articles. mercola.com/sites/articles/archive/2014/03/01/body -electricitygrounding.aspx.

19. Bioinitiative Report: A rationale for Biologically-based Exposure Standards for Low-Intensity Electromagnetic Radiation. 2014-2019; Available from: https://bioinitiative.org/table-ofcontents/.

20. Emerging synergisms between drugs and physiologically-patterned weak magnetic fields: implications for neuropharmacology and the human population in the twenty-first century. Current neuropharmacology, 2007. 5(4): p. 278-288 https:// www.ncbi.nlm.nih.gov/pmc/articles/PMC2644491/

21. Magneto-electric Nanoparticles to Enable Fieldcontrolled High-Specificity Drug Delivery to Eradicate Ovarian Cancer Cells. Nature, 20130 https:// www.nature.com/articles/srep02953

22. Palisano, F.X.H.a.J.R., The Application of Electric Fields in Biology and Medicine. December 20th 2017.

23. Anderson, A. The Man Who Wasn't There: Exploring the science of the self. 26 August 2015; Available from: https://www.newscientist.com/article/mg2273 0360-700-the-man-whowasnt-there-exploring-thescience-of-the-self/\#ixzz69ucLxGpq. 